OPEN ACCESS

Edited by:

Ovidiu Constantin Baltatu, Anhembi Morumbi University, Brazil

Reviewed by: Yasser Mohamed EL-WAZIR, Suez Canal University, Egypt Alexander N. Kapustin, King's College London, UK

${ }^{*}$ Correspondence:

Juan Xu

newxj@hotmail.com

Ximin Guo

guoxim@163.com

Specialty section:

This article was submitted to Integrative Physiology, a section of the journa

Frontiers in Physiology

Received: 06 December 2016 Accepted: 23 February 2017

Published: 08 March 2017

Citation:

Shi Q, Qian Z, Liu D, Sun J, Xu J and Guo X (2017) Maintaining the Phenotype Stability of Chondrocytes Derived from MSCs by C-Type Natriuretic Peptide.

Front. Physiol. 8:143

doi: 10.3389/fphys.2017.00143

\section{Maintaining the Phenotype Stability of Chondrocytes Derived from MSCs by C-Type Natriuretic Peptide}

\author{
Quan Shi ${ }^{1,2}$, Zhiyong Qian ${ }^{2,3}$, Donghua Liü ${ }^{2}$, Jie Sun ${ }^{2,4}$, Juan Xu ${ }^{1 *}$ and Ximin Guo ${ }^{2 *}$ \\ ${ }^{1}$ Department of Stomatology, Chinese People's Liberation Army General Hospital, Beijing, China, ${ }^{2}$ Department of Advanced \\ Interdisciplinary Studies, Institute of Basic Medical Sciences and Tissue Engineering Research Center, Academy of Military \\ Medical Sciences, Beijing, China, ${ }^{3}$ School of Biological Science and Medical Engineering, Beihang University, Beijing, China, \\ ${ }^{4}$ Stomatology Center, General Hospital of Armed Police Forces, Beijing, China
}

Mesenchymal stem cells (MSCs) play a critical role in cartilage tissue engineering. However, MSCs-derived chondrocytes or cartilage tissues are not stable and easily lose the cellular and cartilage phenotype during long-term culture in vitro or implantation in vivo. As a result, chondrocytes phenotypic instability can contribute to accelerated ossification. Thus, it is a big challenge to maintain their correct phenotype for engineering hyaline cartilage. As one member of the natriuretic peptide family, C-type natriuretic peptide (CNP) is found to correlate with the development of the cartilage, affect the chondrocytes proliferation and differentiation. Besides, based on its biological effects on protection of extracellular matrix of cartilage and inhibition of mineralization, we hypothesize that CNP may contribute to the stability of chondrocyte phenotype of MSCs-derived chondrocytes.

Keywords: mesenchymal stem cells, chondrogenic differentiation, phenotype stability, C-type natriuretic peptide, hypothesis

\section{INTRODUCTION}

Cartilage damage caused by the osteoarthritis (OA) can lead to chronic pain and disability. The global prevalence of knee OA was estimated to be 3.8\% (2010), which brings a heavy burden to the society (Cross et al., 2014). Articular cartilage has a limited capacity for spontaneous repair after injury (Savkovic et al., 2014). Cartilage tissue engineering is considered more applicable for articular cartilage repair. Articular chondrocytes (ACs) and mesenchymal stem cells (MSCs; Hubka et al., 2014; Savkovic et al., 2014) are often employed as the seed cells in cartilage engineering. As a native and differentiated cell type, the application of ACs is limited because it is associated with dedifferentiation during expansion in vitro and limited donor tissue supply (Hong and Reddi, 2013; Hubka et al., 2014). MSCs, for example, bone marrow-derived MSCs, or adipose-derived MSCs, have the ability of differentiation into functional chondrocytes under appropriate culture conditions (Mazor et al., 2014). With many advantages, MSCs are widely used in the field of tissue engineering and being put prodigious faith by researchers.

However, accumulated evidences have indicated that the application of MSCs as seed cells for cartilage engineering is not yet as ideal as previously proposed. The chondrocytes and cartilage tissue obtained from MSCs are not stable and will easily lose their cellular and hyaline cartilage tissue phenotype during long time culture in vitro and transplantation in vivo (Tatebe et al., 2005; Pelttari et al., 2006; Farrell et al., 2014; Hubka et al., 2014). These phenotypic instability is characterized by an up-regulated expression of collagen type $\mathrm{X}(\mathrm{Col} \mathrm{X})$, matrix metalloproteinase 
(MMP), and an increase in alkaline phosphatase (ALP) activity, which are all associated with osteogenic differentiation (Hubka et al., 2014). Switch from chondrogenic to osteogenic phenotype of the MSCs-derived cartilage results in loss of the physiological function of articular cartilage. Thus, how to maintain the stability of MSCs-derived chondrocytes phenotype is an important problem that needs to be solved.

Developmentally, transient cartilage is found in the cartilage anlage of endochondral bones (Delgado-Martos et al., 2013), such as the growth plate of long bones of the limbs. In this unique microenvironment, the mesenchymal stem cells undergo a series of special biological processes: condensation, overt differentiation of chondrocytes, proliferation, maturation, hypertrophy, and replacement of chondrocytes by osteoblasts. (Hall and Miyake, 2000; Tuan, 2006; Nguyen et al., 2016). Therefore, if out of the microenvironment of cartilage formation or the microenvironment was damaged, it is likely a natural propensity for the MSCs to differentiate into chondrocytes and then progress to hypertrophy, eventually ossified. In contrast, the chondrocytes in permanent hyaline cartilage on the articular joint surface would not develop to hypertrophy through lifespan. Thus, to make MSCs-derived cartilage as an available clinical therapy, measures must be taken to maintain the phenotype of MSCs-derived chondrocytes at the proliferating or prehypertrophic stage and prevent them from undergoing terminal differentiation to calcified tissue.

In order to solve this problem, some methods have been searched: co-culture of ACs and MSCs (Hubka et al., 2014), Hypoxia (Lee et al., 2013), supplement parathyroid hormonerelated protein (PTHrP; Kim et al., 2008) or activation of TGF $\beta$ pathway (Craft et al., 2015), etc. However, ideal methods for maintaining the phenotype stability of MSCs-derived chondrocytes have not been established and proved clinically translatable, and each of the methods mentioned above has its disadvantages. For example, in order to co-culture of MSCs and ACs, it may require additional surgery and incision; Hypoxia is relatively difficult to implement; PTHrP and TGF $\beta$ pathway have extensive biological functions, which will affect other cells, tissues or organs. Besides, the main purpose of cartilage tissue engineering is to repair cartilage damage caused by OA, so it is important to find an effective solution to maintain the phenotype under the inflammatory microenvironment.

C-type natriuretic peptide (CNP) is one member of the natriuretic peptide family which consists of atrial natriuretic peptide (ANP), brain/B-type natriuretic peptide (BNP) and CNP (Olney, 2006). Different from the other natriuretic peptides, CNP mainly expresses in the growth plate of long bones limbs and plays a critical role in maintaining cartilage homeostasis through its effects on both chondrocyte proliferation and differentiation (Mericq et al., 2000; Prickett et al., 2005; Peake et al., 2014). Data from in vivo and vitro studies show that CNP and its receptor of natriuretic peptide receptor-B (NPR-B) can affect growth of cartilage, chondrogenic differentiation, and mineralization of the cartilage. Genetic mutations in CNP or NPR-B can lead to achondroplasia-like dwarfism in both mice and humans (Chusho et al., 2001; Nakao et al., 2015). CNP can stimulate chondrocytes proliferation and cartilage matrix production, down-regulates the expression of endochondral ossification markers (Waldman et al., 2008) and delay mineralization of tibia (Agoston et al., 2007). Even under the environment of inflammation, CNP can protect the cartilage matrix from degradation (Krejci et al., 2005). Therefore, CNP may play an important role in maintaining the stability of chondrocyte phenotype derived from MSCs.

\section{HYPOTHESIS}

For clinical application, a stable chondrogenic phenotype of MSCs must be achieved. Based on the previous reports, we hypothesize that CNP is potentially a candidate to maintain the stability of chondrogenic phenotype MSCs-derived chondrocytes.

\section{EVALUATION OF THE HYPOTHESIS CNP Promotes the Cartilage Development and Chondrogenic Differentiation}

In mammals, the long bones of limbs are formed through endochondral ossification, which involves the conversion of an initial cartilage template into bone via proliferation, hypertrophy, cell death, and eventually ossified in the growth plate. The mRNA of CNP and NPR-B can be detected in the growth plate (Yamashita et al., 2000; Chusho et al., 2001) and the immunofluorescence also confined this (Olney, 2006). In animal models, mutation or knockout of CNP or NPR-B can lead to dwarfism (Komatsu et al., 2002; Tamura and Garbers, 2003); In contrast, ectopic CNP can rescue growth retardation in mouse model of achondroplasia (Yasoda et al., 2004).

In humans, genetic mutations of NPR-B can result in a disproportionate dwarfism, named acromesomelic dysplasia, Maroteaux type (AMDM) (Bartels et al., 2004). Conversely, skeletal overgrowth has been observed in patients with overexpression of CNP caused by a balanced translocation (Moncla et al., 2007). Moreover, the CNP analog has been used as a therapy for achondroplasia in some clinical trials (Legeai-Mallet, 2016).

In vitro studies have revealed that CNP can promote proliferation of primary chondrocytes (Waldman et al., 2008), as well as chondrocytes-derived from MSCs (Tezcan et al., 2010; Kocamaz et al., 2012). Woods et al. revealed that CNP could regulate cellular condensation of mouse embryonic limbs bud cells during micromass culture by increasing the expression of $\mathrm{N}$-cadherin (Woods et al., 2007). Besides, CNP can stimulate cartilage extracellular matrix deposition. Supplement of $10 \mathrm{nM}$ CNP increased accumulation of proteoglycans and collagen in the culture of chondrocytes (Waldman et al., 2008). By measuring the ${ }^{35} \mathrm{SO}_{4}$, Mericq V et al. found that CNP increased the synthesis of glycosaminoglycan (GAG), one of the main cartilage matrix components (Mericq et al., 2000). Addition of CNP further increased the GAG synthesis in the human trabecular bone derived MSCs (Tezcan et al., 2010) and chicken bone marrow derived MSCs (Kocamaz et al., 2012).

What's more, in rat chondrosarcoma (RCS) chondrocyte model, CNP counteracts fibroblast growth factor 2 (FGF2) effects (which inhibit proliferation and trigger matrix degradation) by inhibiting the Erk pathway at the level of Raf-1. (Krejci et al., 2005; Pejchalova et al., 2007). Besides, CNP can activate protein kinase G (PKG) II and upregulate the synthesis of 
the chondrocyte extracellular matrix through as yet unknown mechanism (Pejchalova et al., 2007). Additionally, increased CNP expression can be stimulated by dexamethasone, which is routinely used for chondrogenic culture of MSCs (Agoston et al., 2006).

\section{CNP Protects Extracellular Matrix of Cartilage}

CNP protects the RCS extracellular matrix from degradation by inhibiting the FGF2-mediated catabolic effects and partially antagonizes FGF2-induced expression, release and activation of MMP2, MMP3, MMP9, MMP10, and MMP13 (Krejci et al., 2005), which are all associated with the degradation of cartilage matrix. Manoj Ramachandran revealed that treatment with CNP can protect the cartilage matrix by reducing the release of nitric oxide (NO) and prostaglandin E2 $\left(\mathrm{PGE}_{2}\right)$ and blocked catabolic effects induced by interleukin-1 $\beta$ (IL-1 $\beta$ ) in a dose-dependent manner (Ramachandran et al., 2011; Peake et al., 2013).

Even in the inflammatory environment, CNP overexpression in chondrocytes can turnover endochondral growth delay and

TABLE 1 | Summary of the positive effects of CNP on maintaining the chondrocytes phenotype stability.

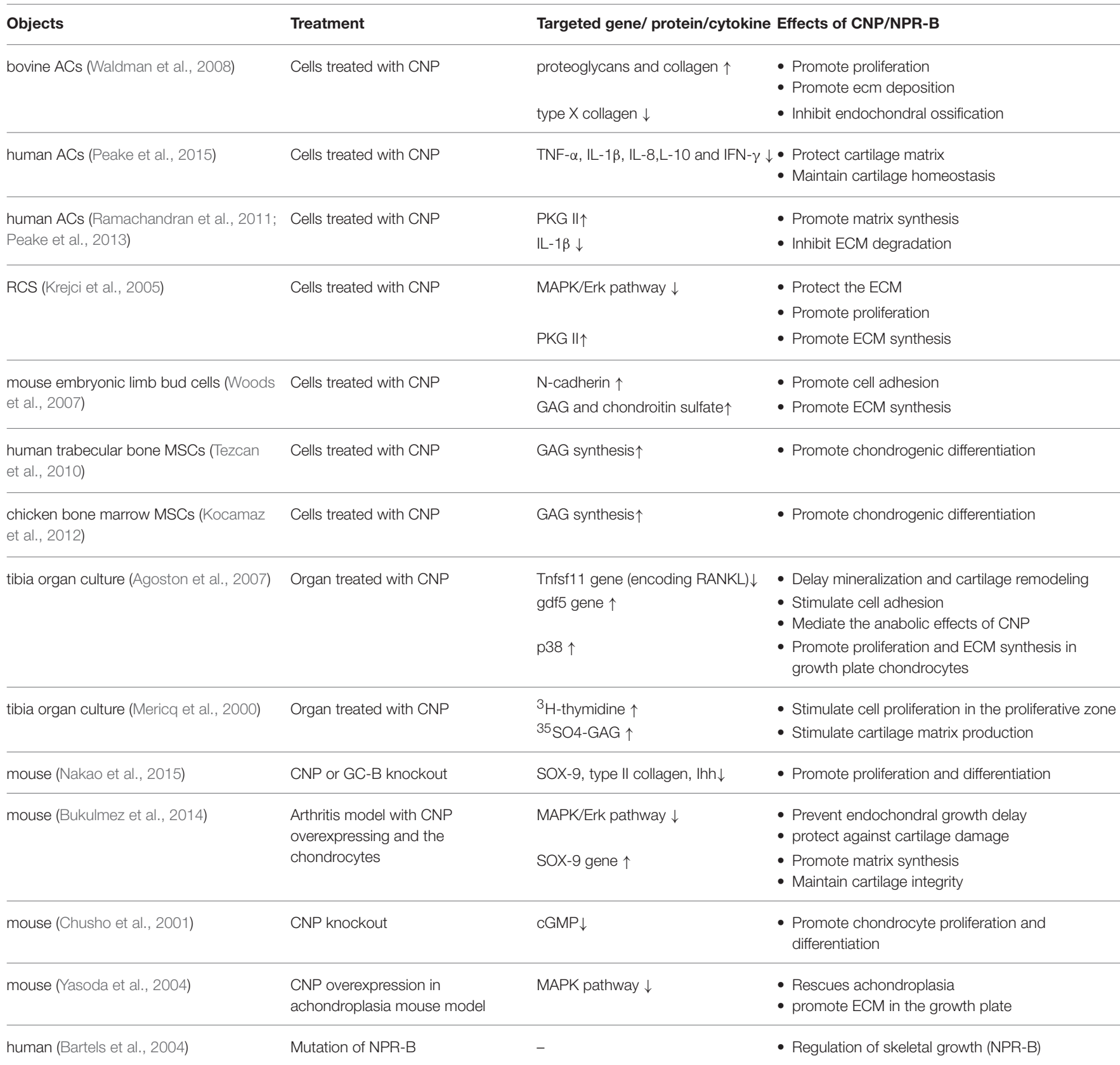

$\uparrow$, up-regulate, promote, activate or increase; $\downarrow$, down-regulate, inhibit, or decrease; RCS, rat chondrosarcoma; ACs, articular chondrocytes; ECM, extracellular matrix; GC-B, guanylyl cyclase-B; Ihh, Indian hedgehog homolog; GAG, glycosaminoglycan.X. 
inhibit cartilage damage in mouse model (Bukulmez et al., 2014). By culture of human chondrocytes in IL-1 $\beta$ conditioned medium in vitro, Peake et al. revealed that CNP can reduce inflammatory factor and hyaluronan production in human chondrocytes via members of the multidrug resistance protein (MRP) and diminish proinflammatory effects, suggesting that the CNP pathway is protective (Peake et al., 2015).

An appropriate microenvironment is important for the physiological activities of the cells. In the growth plate, chondrocytes undergo metabolic changes from proliferating to hypertrophic state and may also transdifferentiate to osteoblast-like cells (Yang et al., 2014). While in the proliferative zone, instead of becoming hypertrophy, or calcify. (Brighton, 1984) This zone composed large amount of extracellular matrix, such as proteoglycans. Besides, it was concluded that very little degradation of the extracellular matrix occurs in the proliferative zone. (Brighton, 1978, 1984) Considering that NPR-B mRNA is expressed primarily in these zones (Olney, 2006), CNP may play a critical role in maintaining the chondrocytes phenotype by balancing the rate of cartilage production of the extracellular matrix of the chondrocytes.

\section{CNP Inhibits Mineralization}

As previously mentioned, Col X, ALP, MMP, and RANKL, etc., are the main markers associated with endochondral ossification. CNP can inhibit endochondral ossification by inhibition of the expression of these factors and maintain the chondrocyte phenotype. In vitro, CNP stimulation decreased the expression of $\mathrm{Col} \mathrm{X}$ in chondrocytes (Waldman et al., 2008). Besides, in an organ culture model, CNP can delay tibia mineralization by down-regulating the Tnfsf11 gene that encodes RANKL (Agoston et al., 2007). RANKL is expressed in hypertrophic cartilage and it can stimulate the removal of hypertrophic cartilage by osteoclasts and facilitate vascular invasion and ossification (Xing et al., 2005). These remodeling events could be delayed after the expressing of RANKL was inhibited. Moreover, CNP may participate in the Indian hedgehog/Parathyroid hormone-related protein (Ihh/PTHrP) loop that inhibits prehypertrophic chondrocytes from entering the hypertrophic phase (Yamashita et al., 2000; Olney, 2006). The positive effects of CNP on maintaining the

\section{REFERENCES}

Agoston, H., Baybayan, L., and Beier, F. (2006). Dexamethasone stimulates expression of C-type natriuretic peptide in chondrocytes. BMC Musculoskelet. Disord. 7:87. doi: 10.1186/1471-2474-7-87

Agoston, H., Khan, S., James, C. G., Gillespie, J. R., Serra, R., Stanton, L. A., et al. (2007). C-type natriuretic peptide regulates endochondral bone growth through p38 MAP kinase-dependent and -independent pathways. BMC Dev. Biol. 7:18. doi: 10.1186/1471-213X-7-18

Bartels, C. F., Bukulmez, H., Padayatti, P., Rhee, D. K., Van Ravenswaaij-Arts, C., Pauli, R. M., et al. (2004). Mutations in the transmembrane natriuretic peptide receptor NPR-B impair skeletal growth and cause acromesomelic dysplasia, type Maroteaux. Am. J. Hum. Genet. 75, 27-34. doi: 10.1086/422013

Brighton, C. T. (1978). Structure and function of the growth plate. Clin. Orthop. Relat. Res. 136, 22-32. doi: 10.1097/00003086-197810000-00003
MSCs-Derived chondrocytes phenotype stability are summarized in Table 1.

\section{CONCLUSION}

Phenotypic instability restricts the application of the MSCs as seed cells in cartilage engineering and an ideal solution has not been established. These previous studies indicate that CNP can promote the MSCs differentiate into chondrocytes at a higher efficiency compared to the traditional methods and it can increase the expression of related chondrogenic markers in a short term. However, none of these papers has long-term in vitro or in vivo studies to observe the effect of CNP on the stability of the MCS-derived chondrocytes/cartilage.

Based on available research results on CNP and MSCs studies and the developmental biology of cartilage and bone tissue, we propose that CNP may represent an important candidate as a regulator to maintain the chondrogenic phenotype of MSCs-derived chondrocytes as well as MSCs-based engineered cartilage both in vitro and in vivo. The potential mechanisms maybe: (1) CNP could down-regulate the expression of RANK and $\mathrm{Col} \mathrm{X}$ to maintain the MSCs derived chondrocytes at the proliferating or prehypertrophic stage. (2) CNP could protect the cartilage matrix and inhibit calcification under the inflammation environment caused by the OA. Once verified, our hypothesis will likely direct the development of phenotypically more stable cartilage tissue for cartilage regeneration.

\section{AUTHOR CONTRIBUTIONS}

Substantial contribution to the concept and design of this study: QS, JX, and XG; literature retrieval and information collection: QS, ZQ, DL, and JS; manuscript drafting: QS; manuscript revising: XG and JX. All authors read and approved the final manuscript.

\section{FUNDING}

This study was supported in part by grants from the National Natural Scientific Foundation of China (NSFC No. 31170926 and No.81170523).

Brighton, C. T. (1984). The growth plate. Orthop. Clin. North Am. 15, 571-595. Bukulmez, H., Khan, F., Bartels, C. F., Murakami, S., Ortiz-Lopez, A., Sattar, A., et al. (2014). Protective effects of C-type natriuretic peptide on linear growth and articular cartilage integrity in a mouse model of inflammatory arthritis. Arthritis Rheumatol. 66, 78-89. doi: 10.1002/art.38199

Chusho, H., Tamura, N., Ogawa, Y., Yasoda, A., Suda, M., Miyazawa, T., et al. (2001). Dwarfism and early death in mice lacking C-type natriuretic peptide. Proc. Natl. Acad. Sci. U.S.A. 98, 4016-4021. doi: 10.1073/pnas.071389098

Craft, A. M., Rockel, J. S., Nartiss, Y., Kandel, R. A., Alman, B. A., and Keller, G. M. (2015). Generation of articular chondrocytes from human pluripotent stem cells. Nat. Biotechnol. 33, 638-645. doi: 10.1038/nbt.3210

Cross, M., Smith, E., Hoy, D., Nolte, S., Ackerman, I., Fransen, M., et al. (2014). The global burden of hip and knee osteoarthritis: estimates from the global burden of disease 2010 study. Ann. Rheum. Dis. 73, 1323-1330. doi: 10.1136/annrheumdis-2013-204763 
Delgado-Martos, M. J., Touza Fernandez, A., Canillas, F., Quintana-Villamandos, B., Santos Del Riego, S., Delgado-Martos, E., et al. (2013). Does the epiphyseal cartilage of the long bones have one or two ossification fronts? Med. Hypotheses 81, 695-700. doi: 10.1016/j.mehy.2013.07.029

Farrell, M. J., Fisher, M. B., Huang, A. H., Shin, J. I., Farrell, K. M., and Mauck, R. L. (2014). Functional properties of bone marrow-derived MSC-based engineered cartilage are unstable with very long-term in vitro culture. J. Biomech. 47, 2173-2182. doi: 10.1016/j.jbiomech.2013.10.030

Hall, B. K., and Miyake, T. (2000). All for one and one for all: condensations and the initiation of skeletal development. Bioessays 22, 138-147. doi: 10.1002/(SICI)1521-1878(200002)22:2<138::AID-BIES5>3.0.CO;2-4

Hong, E., and Reddi, A. H. (2013). Dedifferentiation and redifferentiation of articular chondrocytes from surface and middle zones: changes in microRNAs221/-222, -140, and -143/145 expression. Tissue Eng. Part A 19, 1015-1022. doi: 10.1089/ten.tea.2012.0055

Hubka, K. M., Dahlin, R. L., Meretoja, V. V., Kasper, F. K., and Mikos, A. G. (2014). Enhancing chondrogenic phenotype for cartilage tissue engineering: monoculture and coculture of articular chondrocytes and mesenchymal stem cells. Tissue Eng. Part B Rev. 20, 641-654. doi: 10.1089/ten.teb.2014.0034

Kim, Y. J., Kim, H. J., and Im, G. I. (2008). PTHrP promotes chondrogenesis and suppresses hypertrophy from both bone marrow-derived and adipose tissue-derived MSCs. Biochem. Biophys. Res. Commun. 373, 104-108. doi: 10.1016/j.bbrc.2008.05.183

Kocamaz, E., Gok, D., Cetinkaya, A., and Tufan, A. C. (2012). Implication of C-type natriuretic peptide-3 signaling in glycosaminoglycan synthesis and chondrocyte hypertrophy during TGF-betal induced chondrogenic differentiation of chicken bone marrow-derived mesenchymal stem cells. $J$. Mol. Histol. 43, 497-508. doi: 10.1007/s10735-012-9430-2

Komatsu, Y., Chusho, H., Tamura, N., Yasoda, A., Miyazawa, T., Suda, M., et al. (2002). Significance of C-type natriuretic peptide (CNP) in endochondral ossification: analysis of CNP knockout mice. J. Bone Miner. Metab. 20, 331-336. doi: $10.1007 /$ s007740200048

Krejci, P., Masri, B., Fontaine, V., Mekikian, P. B., Weis, M., Prats, H., et al. (2005). Interaction of fibroblast growth factor and $\mathrm{C}$-natriuretic peptide signaling in regulation of chondrocyte proliferation and extracellular matrix homeostasis. J. Cell Sci. 118, 5089-5100. doi: 10.1242/jcs.02618

Lee, H. H., Chang, C. C., Shieh, M. J., Wang, J. P., Chen, Y. T., Young, T. H., et al. (2013). Hypoxia enhances chondrogenesis and prevents terminal differentiation through PI3K/Akt/FoxO dependent anti-apoptotic effect. Sci. Rep. 3:2683. doi: $10.1038 /$ srep02683

Legeai-Mallet, L. (2016). C-type natriuretic peptide analog as therapy for achondroplasia. Endocr. Dev. 30, 98-105. doi: 10.1159/000439334

Mazor, M., Lespessailles, E., Coursier, R., Daniellou, R., Best, T. M., and Toumi, H. (2014). Mesenchymal stem-cell potential in cartilage repair: an update. J. Cell. Mol. Med. 18, 2340-2350. doi: 10.1111/jcmm.12378

Mericq, V., Uyeda, J. A., Barnes, K. M., De Luca, F., and Baron, J. (2000). Regulation of fetal rat bone growth by C-type natriuretic peptide and cGMP. Pediatr. Res. 47, 189-193. doi: 10.1203/00006450-200002000-00007

Moncla, A., Missirian, C., Cacciagli, P., Balzamo, E., Legeai-Mallet, L., Jouve, J. L., et al. (2007). A cluster of translocation breakpoints in $2 \mathrm{q} 37$ is associated with overexpression of NPPC in patients with a similar overgrowth phenotype. Hum. Mutat. 28, 1183-1188. doi: 10.1002/humu.20611

Nakao, K., Osawa, K., Yasoda, A., Yamanaka, S., Fujii, T., Kondo, E., et al. (2015). The Local CNP/GC-B system in growth plate is responsible for physiological endochondral bone growth. Sci. Rep. 5:10554. doi: 10.1038/srep10554

Nguyen, T. D., Oloyede, A., Singh, S., and Gu, Y. (2016). Investigation of the effects of extracellular osmotic pressure on morphology and mechanical properties of individual chondrocyte. Cell Biochem. Biophys. 74, 229-240. doi: 10.1007/ s12013-016-0721-1

Olney, R. C. (2006). C-type natriuretic peptide in growth: a new paradigm. Growth Horm. IGF Res. 16(Suppl. A), S6-S14. doi: 10.1016/j.ghir.2006.03.016

Peake, N. J., Bader, D. L., Vessillier, S., Ramachandran, M., Salter, D. M., Hobbs, A. J., et al. (2015). C-type natriuretic peptide signalling drives homeostatic effects in human chondrocytes. Biochem. Biophys. Res. Commun. 465, 784-789. doi: 10.1016/j.bbrc.2015.08.087

Peake, N. J., Hobbs, A. J., Pingguan-Murphy, B., Salter, D. M., Berenbaum, F., and Chowdhury, T. T. (2014). Role of C-type natriuretic peptide signalling in maintaining cartilage and bone function. Osteoarthr. Cartil. 22, 1800-1807. doi: 10.1016/j.joca.2014.07.018
Peake, N., Su, N., Ramachandran, M., Achan, P., Salter, D. M., Bader, D. L., et al. (2013). Natriuretic peptide receptors regulate cytoprotective effects in a human ex vivo 3D/bioreactor model. Arthritis Res. Ther. 15:R76. doi: 10.1186/ $\operatorname{ar} 4253$

Pejchalova, K., Krejci, P., and Wilcox, W. R. (2007). C-natriuretic peptide: an important regulator of cartilage. Mol. Genet. Metab. 92, 210-215. doi: 10.1016/j.ymgme.2007.06.014

Pelttari, K., Winter, A., Steck, E., Goetzke, K., Hennig, T., Ochs, B. G., et al. (2006). Premature induction of hypertrophy during in vitro chondrogenesis of human mesenchymal stem cells correlates with calcification and vascular invasion after ectopic transplantation in SCID mice. Arthritis Rheum. 54, 3254-3266. doi: 10.1002/art.22136

Prickett, T. C., Lynn, A. M., Barrell, G. K., Darlow, B. A., Cameron, V. A., Espiner, E. A., et al. (2005). Amino-terminal proCNP: a putative marker of cartilage activity in postnatal growth. Pediatr. Res. 58, 334-340. doi: 10.1203/01.PDR.0000169964.66260.4B

Ramachandran, M., Achan, P., Salter, D. M., Bader, D. L., and Chowdhury, T. T. (2011). Biomechanical signals and the C-type natriuretic peptide counteract catabolic activities induced by IL-1beta in chondrocyte/agarose constructs. Arthritis Res. Ther. 13:R145. doi: 10.1186/ar3459

Savkovic, V., Li, H., Seon, J. K., Hacker, M., Franz, S., and Simon, J. C. (2014). Mesenchymal stem cells in cartilage regeneration. Curr. Stem Cell Res. Ther. 9, 469-488. doi: 10.2174/1574888X09666140709111444

Tamura, N., and Garbers, D. L. (2003). Regulation of the guanylyl cyclaseB receptor by alternative splicing. J. Biol. Chem. 278, 48880-48889. doi: $10.1074 /$ jbc.M308680200

Tatebe, M., Nakamura, R., Kagami, H., Okada, K., and Ueda, M. (2005). Differentiation of transplanted mesenchymal stem cells in a large osteochondral defect in rabbit. Cytotherapy 7, 520-530. doi: 10.1080/146532405003 61350

Tezcan, B., Serter, S., Kiter, E., and Tufan, A. C. (2010). Dose dependent effect of C-type natriuretic peptide signaling in glycosaminoglycan synthesis during TGF-betal induced chondrogenic differentiation of mesenchymal stem cells. J. Mol. Histol. 41, 247-258. doi: 10.1007/s10735-010-9284-4

Tuan, R. S. (2006). Stemming cartilage degeneration: adult mesenchymal stem cells as a cell source for articular cartilage tissue engineering. Arthritis Rheum. 54, 3075-3078. doi: 10.1002/art.22148

Waldman, S. D., Usmani, Y., Tse, M. Y., and Pang, S. C. (2008). Differential effects of natriuretic peptide stimulation on tissue-engineered cartilage. Tissue Eng. Part A 14, 441-448. doi: 10.1089/tea.2007.0035

Woods, A., Khan, S., and Beier, F. (2007). C-type natriuretic peptide regulates cellular condensation and glycosaminoglycan synthesis during chondrogenesis. Endocrinology 148, 5030-5041. doi: 10.1210/en.2007-0695

Xing, L., Schwarz, E. M., and Boyce, B. F. (2005). Osteoclast precursors, RANKL/RANK, and immunology. Immunol. Rev. 208, 19-29. doi: 10.1111/j.0105-2896.2005.00336.x

Yamashita, Y., Takeshige, K., Inoue, A., Hirose, S., Takamori, A., and Hagiwara, H. (2000). Concentration of mRNA for the natriuretic peptide receptor-C in hypertrophic chondrocytes of the fetal mouse tibia. J. Biochem. 127, 177-179. doi: 10.1093/oxfordjournals.jbchem.a022591

Yang, L., Tsang, K. Y., Tang, H. C., Chan, D., and Cheah, K. S. (2014). Hypertrophic chondrocytes can become osteoblasts and osteocytes in endochondral bone formation. Proc. Natl. Acad. Sci. U.S.A. 111, 12097-12102. doi: 10.1073/pnas.1302703111

Yasoda, A., Komatsu, Y., Chusho, H., Miyazawa, T., Ozasa, A., Miura, M., et al. (2004). Overexpression of CNP in chondrocytes rescues achondroplasia through a MAPK-dependent pathway. Nat. Med. 10, 80-86. doi: $10.1038 / \mathrm{nm} 971$

Conflict of Interest Statement: The authors declare that the research was conducted in the absence of any commercial or financial relationships that could be construed as a potential conflict of interest.

Copyright (C) 2017 Shi, Qian, Liu, Sun, Xu and Guo. This is an open-access article distributed under the terms of the Creative Commons Attribution License (CC BY). The use, distribution or reproduction in other forums is permitted, provided the original author(s) or licensor are credited and that the original publication in this journal is cited, in accordance with accepted academic practice. No use, distribution or reproduction is permitted which does not comply with these terms. 